\title{
Stability analysis of multiple nonequilibrium fixed points in self-consistent electron transport calculations
}

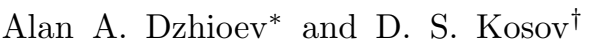 \\ Department of Physics, Université Libre de Bruxelles, Campus Plaine, \\ CP 231, Blvd du Triomphe, B-1050 Brussels, Belgium
}

\begin{abstract}
We present a method to perform stability analysis of nonequilibrium fixed points appearing in self-consistent electron transport calculations. The nonequilibrium fixed points are given by the self-consistent solution of stationary, nonlinear kinetic equation for single-particle density matrix. We obtain the stability matrix by linearizing the kinetic equation around the fixed points and analyze the real part of its spectrum to assess the asymptotic time behavior of the fixed points. We derive expressions for the stability matrices within Hartree-Fock and linear response adiabatic time-dependent density functional theory. The stability analysis of multiple fixed points is performed within the nonequilibrium Hartree-Fock approximation for the electron transport through a molecule with a spin-degenerate single level with local Coulomb interaction.
\end{abstract}

\section{INTRODUCTION}

The existence of nonunique steady state for nonequilibrium systems of correlated quantum or classical particles is an interesting and open fundamental problem. 1, 2] Multiple steady states in nanojunctions lead to bistabilities and hysteresis loops in the current-voltage characteristics and this currently is a much debated theoretical issue. 3 5] Density functional theory (DFT) and Hartree-Fock based nonequilibrium Green's functions (NEGF) electron transport calculations, which are widely used nowadays, solve nonlinear system of equations for nonequilibrium electron density via selfconsistent iterations. [6 9] Such kind of nonlinear problems may have multiple solutions. [5, [10] In equilibrium case the correct physical solution corresponds to a minimum of the ground state energy. The situation is less clear in nonequilibrium where the system is open and the minimum energy arguments are not applicable anymore. In this paper, we discuss the appearance of multiple fixed points and present a method to eliminate unphysical solutions of steady-state nonequilibrium self-consistent electron transport problem.

Let us consider a finite quantum system (e.g., a molecule) connected to two macroscopic particle reservoirs or thermal baths (e.g., metal electrodes). By projecting out bath degrees of freedom we obtain the kinetic equation for the reduced density matrix $\rho(t)$ of the embedded system

$$
i \frac{d}{d t} \rho(t)=\mathcal{L} \rho(t)
$$

where $\mathcal{L}$ is a non-Hermitian Liouvillian. We assume that the dynamics is markovian and the system is autonomous, i.e. $\mathcal{L}$ does not depend explicitly on time. In

\footnotetext{
*Permanent address: Bogoliubov Laboratory of Theoretical Physics, Joint Institute for Nuclear Research, RU-141980 Dubna, Russia

${ }^{\dagger}$ Electronic address: dkosov@ulb.ac.be
}

this paper we will focus on nonequilibrium steady state. Like an equilibrium represents stationary state of a closed system, a nonequilibrium steady state is the stable, timeinvariant state of an open system. If the dynamics generated by $\mathcal{L}$ is linear, then a nonequilibrium steady state can be unambiguously defined as a state when the left side of the equation (1) becomes zero. However, often times for practical calculations we involve the mean-field approximation (Hartree-Fock or DFT) and the Liouvillian becomes a functional of the reduced density matrix

$$
\mathcal{L}=\mathcal{L}[\rho]
$$

Therefore the kinetic equation (11) becomes nonlinear and the issue of stability of the solution becomes pivotal.

Let us give a few formal definitions from the theory of dynamical dissipative systems, [11, 12] which are relevant to electron transport problem. The density matrices $\bar{\rho}$ at which

$$
\mathcal{L}[\bar{\rho}] \bar{\rho}=0
$$

are called the fixed points of the system. Since Eq. (3) is nonlinear, it generally has multiple solutions, which may or may not be steady state (i.e. stable fixed point). To understand whether or not the fixed point is stable we need to perform stability analysis commonly used for dynamical nonlinear systems. We expand the density around the fixed point

$$
\rho(t)=\bar{\rho}+\delta \rho(t)
$$

and linearize the Liouville equation

$$
\frac{d}{d t} \delta \rho(t)=A \delta \rho(t)
$$

where

$$
A=-i\left[\mathcal{L}[\bar{\rho}]+\left.\frac{\delta \mathcal{L}}{\delta \rho}\right|_{\rho=\bar{\rho}} \bar{\rho}\right]
$$

is the stability matrix. If $A$ is a so-called Hurwitz matrix, i.e., if all eigenvalues $\lambda_{i}$ of $A$ satisfies the conditions 
$\operatorname{Re}\left(\lambda_{i}\right)<0$, then the fixed point $\bar{\rho}$ is asymptotically stable and it is the true steady state of the system. If at least one eigenvalue has positive real part, the solution is unstable and can not be a steady state, since even an infinitesimally small variation of the fixed point density matrix drives the solution away from the fixed point exponentially in time. In our paper we demonstrate that there are several fixed points and multiple steady states in some rather typical cases of electronic transport calculations.

The rest of the paper is organized as follows. In Section II, we discuss nonequilibrium fixed points, linearization and stability matrix for self-consistent electron transport problem and obtain the general expression for the stability matrix. In section III, we apply the method to out of equilibrium Anderson model and demonstrates that it has multiple (stable and unstable) fixed points. Conclusions are given in Section IV. In Appendix A, we derive the kinetic equation for the reduced density matrix of the embedded system. The adiabatic time-dependent KohnSham DFT expressions for stationary Fock matrix and its fluctuating part are given in ppendix B. We use natural units throughout the paper: $\hbar=k_{B}=|e|=1$, where $-|e|$ is the electron charge.

\section{NONEQUILIBRIUM FIXED POINTS, LINEARIZATION AND STABILITY MATRIX}

Let us consider a molecule connected to two electrodes. We partition the system into five parts: the molecule itself, the left/right macroscopically large leads (environment), and the left/right finite buffer zones between the molecule and the environment. The Hamiltonian is written in the following form:

$$
\mathcal{H}=H_{M}+H_{E}+H_{B}+H_{M B}+H_{E B}
$$

The environment and the buffer zones are described by the noninteracting Hamiltonians

$$
\begin{aligned}
& H_{E}=\sum_{\sigma, k \in L, R} \varepsilon_{k} a_{k \sigma}^{\dagger} a_{k \sigma}, \\
& H_{B}=\sum_{\sigma, b \in L, R} \varepsilon_{b} a_{b \sigma}^{\dagger} a_{b \sigma} .
\end{aligned}
$$

Here $\varepsilon_{k}$ denote the continuum single-particle spectra of the left $(k \in L)$ and right $(k \in R)$ lead states, $a_{k \sigma}^{\dagger}\left(a_{k \sigma}\right)$ create (annihilate) electron with spin $\sigma=\uparrow, \downarrow$ in the lead state $k$. The buffer zones have discrete energy spectrum $\varepsilon_{b}$ with corresponding creation and annihilation operators $a_{b \sigma}^{\dagger}$ and $a_{b \sigma}$.

The molecular Hamiltonian is taken in the most general form and contains electronic kinetic energy, electronion interaction, and Coulomb interaction between elec- trons:

$$
H_{M}=\sum_{i j \sigma} T_{i j} a_{i \sigma}^{\dagger} a_{j \sigma}+\frac{1}{2} \sum_{i j m n} \sum_{\sigma \sigma^{\prime}}(i j \mid m n) a_{i \sigma}^{\dagger} a_{m \sigma^{\prime}}^{\dagger} a_{n \sigma^{\prime}} a_{j \sigma},
$$

where

$$
T_{n m}=\int d \mathbf{r} \phi_{n}(\mathbf{r})\left(-\frac{1}{2} \nabla^{2}+V_{\mathrm{e}-\mathrm{i}}(\mathbf{r})\right) \phi_{m}(\mathbf{r})
$$

and

$$
(i j \mid m n)=\int d \mathbf{r} d \mathbf{r}^{\prime} \phi_{i}(\mathbf{r}) \phi_{j}(\mathbf{r}) \frac{1}{\left|\mathbf{r}-\mathbf{r}^{\prime}\right|} \phi_{m}\left(\mathbf{r}^{\prime}\right) \phi_{n}\left(\mathbf{r}^{\prime}\right)
$$

are matrix elements computed in some orthogonal real basis $\left\langle\phi_{i} \mid \phi_{j}\right\rangle=\delta_{i j}$. Here $a_{i \sigma}^{\dagger}$ and $a_{i \sigma}$ are creation and annihilation operators for electron with spin $\sigma$ in molecular state $\left|\phi_{i}\right\rangle$. Hereinafter, the index $b$ refers to discrete single-particle states in either left or right buffer zones, whereas the indices $i, j, m, n$ represent states in the molecular space.

The buffer-environment and molecule-buffer coupling have the standard tunneling form:

$$
\begin{gathered}
H_{E B}=\sum_{\sigma, b k \in L}\left(v_{b k} a_{b \sigma}^{\dagger} a_{k \sigma}+\text { h.c. }\right)+\sum_{\sigma, b k \in R}\left(v_{b k} a_{b \sigma}^{\dagger} a_{k \sigma}+\text { h.c. }\right), \\
H_{M B}=\sum_{i \sigma, b \in L, R}\left(t_{i b \sigma} a_{b \sigma}^{\dagger} a_{i \sigma}+\text { h.c. }\right) .
\end{gathered}
$$

The Liouville equation for the total density matrix $\chi(t)$ is:

$$
i \dot{\chi}(t)=[\mathcal{H}, \chi(t)]
$$

If we project out the environment degrees of freedom and make the standard assumptions (Born-Markov and rotating wave approximations [13] - the details of the derivation are shown in Appendix A) we get the following kinetic equation

$$
i \dot{\rho}(t)=[H, \rho(t)]+\hat{\Pi} \rho(t)
$$

for the reduced density matrix for the embedded molecule (i.e. for the molecule and the buffer zones)

$$
\rho(t)=\operatorname{Tr}_{E} \chi(t) .
$$

Here the Hamiltonian $H$ includes the Lamb shift of the single-particle levels of the buffer zones

$$
H=H_{M}+H_{M B}+\sum_{\sigma, b}\left(\varepsilon_{b}+\Delta_{b}\right) a_{b \sigma}^{\dagger} a_{b \sigma}
$$

and the non-Hermitian dissipator is given by standard Lindblad form

$$
\hat{\Pi} \rho(t)=\sum_{\sigma, b} \sum_{\mu=1,2}\left(2 L_{b \sigma \mu} \rho(t) L_{b \sigma \mu}^{\dagger}-\left\{L_{b \sigma \mu}^{\dagger} L_{b \sigma \mu}, \rho(t)\right\}\right)
$$


with the following Lindblad operators

$$
L_{b \sigma 1}=\sqrt{\gamma_{b}\left(1-f_{b}\right)} a_{b \sigma}, \quad L_{b \sigma 2}=\sqrt{\gamma_{b} f_{b}} a_{b \sigma}^{\dagger} .
$$

Here $\Delta_{b}$ and $\gamma_{b}$ are real and imaginary parts of the standard environment self energy $\sum_{k}\left|v_{b k}\right|^{2} /\left(\varepsilon_{b}-\varepsilon_{k}+i 0^{+}\right)$ and $f_{b \in L / R}=\left[1+e^{\beta_{L / R}\left(\varepsilon_{b}-\mu_{L / R}\right)}\right]^{-1}$.

We emphasize that our kinetic equation (16) does not employ the second order perturbative treatment of molecule-electrode coupling (14), but rather it is the second-order in terms of the coupling between the buffer zone and the environment (13). We have recently demonstrated that for the steady state electron transport calculations the kinetic equation (16) can be made as accurate and exact as necessary in practical calculations by increasing the density of single-particle buffer states $b$ included into the Hamiltonian. 14, 15] The similar idea of the buffer zone between the molecule and the environment has been recently proposed in dynamical simulations of inelastic electron transport. [16]

Let us now consider time-evolution of the expectation value of an arbitrary operator $O$ :

$$
\langle O\rangle_{t}=\operatorname{Tr}[\rho(t) O] .
$$

Using Eq. (16) we obtain

$$
\begin{aligned}
& \frac{d}{d t}\langle O\rangle_{t}=-i\langle[O, H]\rangle_{t} \\
+ & \sum_{\sigma b} \sum_{\mu=1,2}\left(2\left\langle L_{b \sigma \mu}^{\dagger} O L_{b \sigma \mu}\right\rangle_{t}-\left\langle\left\{L_{b \sigma \mu}^{\dagger} L_{b \sigma \mu}, O\right\}\right\rangle_{t}\right) .
\end{aligned}
$$

We apply the time-dependent Hartree-Fock approximation to the two-particle interaction in the molecular Hamiltonian

$$
H_{M}^{(0)}=\sum_{\sigma i j} F_{i j}^{\sigma}(t) a_{i \sigma}^{\dagger} a_{j \sigma}
$$

Here $F_{n m}^{\sigma}(t)$ is the time-dependent Fock matrix

$$
F_{n m}^{\sigma}(t)=T_{n m}+\sum_{i j}\left[(n m \mid i j) P_{i j}(t)-(n i \mid m j) P_{i j}^{\sigma}(t)\right],
$$

which depends on the single-particle density matrices

$$
P_{i j}^{\sigma}(t)=\left\langle a_{j \sigma}^{\dagger} a_{i \sigma}\right\rangle_{t}, \quad P_{i j}(t)=\sum_{\sigma} P_{i j}^{\sigma}(t)
$$

The corresponding expression for the Fock matrix in adiabatic time-dependent Kohn-Sham DFT is given in Appendix B. The Lindblad equation (16) becomes the timedependent Hartree-Fock equation for the nonequilibrium open quantum system, when the full many-body molecular Hamiltonian is approximated by $H_{M}^{(0)}$ (23). We introduce two additional single-particle density matrices:

$$
P_{b j}^{\sigma}(t)=\left\langle a_{j \sigma}^{\dagger} a_{b \sigma}\right\rangle_{t}, \quad P_{b b^{\prime}}^{\sigma}(t)=\left\langle a_{b^{\prime} \sigma}^{\dagger} a_{b \sigma}\right\rangle_{t}
$$

and $P_{j b}^{\sigma}(t)=\left(P_{b j}^{\sigma}(t)\right)^{*}$. By means of (22) we get the close set of time evolution equations for single-particle density matrices

$$
\begin{aligned}
i \frac{d}{d t} P_{i j}^{\sigma}(t) & =\sum_{n}\left[F_{i n}^{\sigma}(t) P_{n j}^{\sigma}(t)-F_{n j}^{\sigma}(t) P_{i n}^{\sigma}(t)\right]+\sum_{b}\left[t_{i b \sigma} P_{b j}^{\sigma}(t)-t_{j b \sigma}^{*} P_{i b}^{\sigma}(t)\right], \\
i \frac{d}{d t} P_{b i}^{\sigma}(t) & =E_{b} P_{b i}^{\sigma}(t)-\sum_{j} F_{j i}^{\sigma}(t) P_{b j}^{\sigma}(t)-\sum_{b^{\prime}} t_{i b^{\prime} \sigma}^{*} P_{b^{\prime} b}^{\sigma}(t)+\sum_{j} t_{j b \sigma}^{*} P_{j i}^{\sigma}(t), \\
i \frac{d}{d t} P_{b b^{\prime}}^{\sigma}(t) & =\left(E_{b}-E_{b^{\prime}}^{*}\right) P_{b b^{\prime}}^{\sigma}(t)-\sum_{i}\left[t_{i b^{\prime} \sigma} P_{b i}^{\sigma}(t)-t_{i b \sigma}^{*} P_{i b^{\prime}}^{\sigma}(t)\right]+2 i \delta_{b b^{\prime}} f_{b} \gamma_{b}
\end{aligned}
$$

with $E_{b}=\varepsilon_{b}-i \gamma_{b}$. Here we include the Lamb shift $\Delta_{b}$ into single-particle energy $\varepsilon_{b}$. These equations of motion are nonlinear because the Fock matrix $F_{i j}^{\sigma}(t)$ depends on the density matrix $P_{i j}^{\sigma}(t)$.

Setting the left side of equations (27) to zero, we obtain the stationary Hartree-Fock equations for nonequilibrium fixed points. These fixed points correspond to stationary single-particle densities, $\bar{P}_{i j}^{\sigma}, \bar{P}_{b i}^{\sigma}, \bar{P}_{i b}^{\sigma}$, and $\bar{P}_{b b^{\prime}}^{\sigma}$, which may or may not be steady state densities. To deter- mine if these fixed points are asymptotically stable, we linearize Eq. (27) around each fixed point. Substituting (here indices $\alpha, \beta$ run over molecular and buffer single particle states)

$$
P_{\alpha \beta}^{\sigma}(t)=\bar{P}_{\alpha \beta}^{\sigma}+\delta P_{\alpha \beta}^{\sigma}(t)
$$

into Eq. (27) and retaining only the terms linear in $\delta P_{\alpha \beta}^{\sigma}(t)$ we get 


$$
\begin{aligned}
i \frac{d}{d t} \delta P_{i j}^{\sigma}(t) & =\sum_{n}\left[\bar{F}_{i n}^{\sigma} \delta P_{n j}^{\sigma}(t)-\bar{F}_{n j}^{\sigma} \delta P_{i n}^{\sigma}(t)+\delta F_{i n}^{\sigma}(t) \bar{P}_{n j}^{\sigma}-\delta F_{n j}^{\sigma}(t) \bar{P}_{i n}^{\sigma}\right]+\sum_{b}\left[t_{i b \sigma} \delta P_{b j}^{\sigma}(t)-t_{j b \sigma}^{*} \delta P_{i b}^{\sigma}(t)\right] \\
i \frac{d}{d t} \delta P_{b i}^{\sigma}(t) & =E_{b} \delta P_{b i}^{\sigma}(t)-\sum_{j}\left[\bar{F}_{j i} \delta P_{b j}^{\sigma}(t)+\delta F_{j i}^{\sigma}(t) \bar{P}_{b j}^{\sigma}\right]-\sum_{b^{\prime}} t_{i b^{\prime} \sigma}^{*} \delta P_{b^{\prime} b}^{\sigma}(t)+\sum_{j} t_{j b \sigma}^{*} \delta P_{j i}^{\sigma}(t), \\
i \frac{d}{d t} \delta P_{b b^{\prime}}^{\sigma}(t) & =\left(E_{b}-E_{b^{\prime}}^{*}\right) \delta P_{b b^{\prime}}^{\sigma}(t)-\sum_{i}\left[t_{i b^{\prime} \sigma} \delta P_{b i}^{\sigma}(t)-t_{i b \sigma}^{*} \delta P_{i b^{\prime}}^{\sigma}(t)\right] .
\end{aligned}
$$

Here the Fock matrix at the fixed point is

$$
\bar{F}_{n m}^{\sigma}=T_{n m}+\sum_{i j}\left[(n m \mid i j) \bar{P}_{i j}-(n i \mid m j) \bar{P}_{i j}^{\sigma}\right] .
$$

and its time-dependent fluctuation around this fixed point is

$$
\delta F_{n m}^{\sigma}(t)=\sum_{i j}\left[(n m \mid i j) \delta P_{i j}(t)-(n i \mid m j) \delta P_{i j}^{\sigma}(t)\right] .
$$

The adiabatic time-dependent Kohn-Sham DFT expressions for stationary Fock matrix and its fluctuating part are given in appendix B.

The system of equations (29) can be rewritten as the set of linear differential equation

$$
\frac{d}{d t} \delta P_{\alpha \beta}^{\sigma}(t)=\sum_{\alpha^{\prime} \beta^{\prime}} \sum_{\sigma^{\prime}} A_{\alpha \beta}^{\sigma \sigma^{\prime}, \alpha^{\prime} \beta^{\prime}} \delta P_{\alpha^{\prime} \beta^{\prime}}^{\sigma^{\prime}}(t) .
$$

Here $A_{\alpha \beta, \alpha^{\prime} \beta^{\prime}}^{\sigma \sigma^{\prime}}$ is the spin-dependent stability matrix. Each element of this stability matrix can be readily obtained from (29). In general case it is sparse, complex and non-Hermitian matrix. Now we need to find eigenvalues $\lambda_{i}$ of the stability matrix $A_{\alpha \beta}^{\sigma \sigma^{\prime}}, \alpha^{\prime} \beta^{\prime}$ and analyze their real parts. If $\operatorname{Re}\left(\lambda_{i}\right)<0$ for all eigenvalues in the spectrum, then the fixed point $\bar{P}_{\alpha \beta}^{\sigma}$ is asymptotically stable as $t \rightarrow \infty$ and, therefore, it is the true steady state of the system. If at least one eigenvalue has a positive real part, the solution becomes unstable along this mode and it is not a steady state. [11, 12] The purely imaginary eigenvalues of stability matrix correspond to the periodically oscillating fixed points which may be relevant to dynamical picture of Coulomb blockade regime. [17] If one of $\lambda_{i}$ becomes zero, the system undergoes zero-eigenvalue bifurcation which can be saddle-node, transcritical, or pitchfork type bifurcation. 12]

\section{EXAMPLE CALCULATIONS}

To illustrate the theory we consider electron transport through a molecule with a spin-degenerate single level with local Coulomb interaction (so called Anderson model). The Hamiltonian is given by

$$
H=H_{M}-\sum_{\sigma b} t_{b}\left(a_{\sigma}^{\dagger} a_{b \sigma}+h . c\right)+\sum_{\sigma b} \varepsilon_{b} a_{b \sigma}^{\dagger} a_{b \sigma},
$$

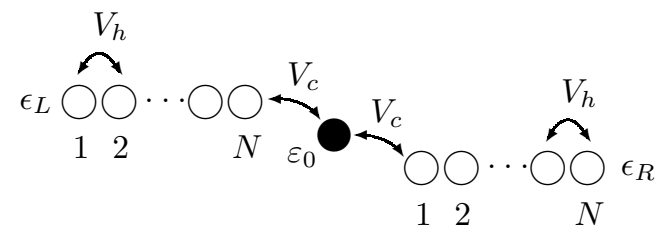

Left buffer $\quad$ Right buffer

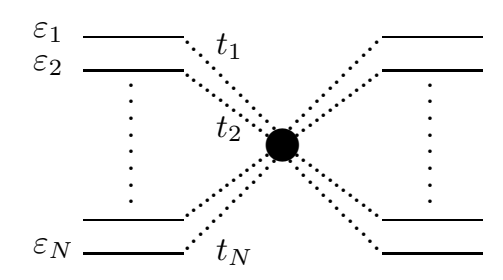

FIG. 1: Schematic illustration of the model system used in the electron transport calculations. In the upper part of the figure only the first left $\epsilon_{1}(1 \in L)$ and the last right $\epsilon_{N}(N \in R)$ atoms from the buffer zones are attached to the environment. After the diagonalization of the buffer zone Hamiltonian, each energy level $\varepsilon_{b}$ is connected to the dissipators and the molecule (lower part of the figure).

where the molecular Hamiltonian is

$$
H_{M}=\varepsilon_{0} \sum_{\sigma} a_{\sigma}^{\dagger} a_{\sigma}+U a_{\uparrow}^{\dagger} a_{\uparrow} a_{\downarrow}^{\dagger} a_{\downarrow} .
$$

In our calculations left and right buffer zones are modeled as a finite chain of $N$ atoms, characterized by the hopping parameter $V_{h}$ and the on-site energy $\epsilon_{L, R}$ (Fig. (1). Thus, the energy spectrum of the each buffer is given by

$$
\varepsilon_{b}=\epsilon_{L, R}+2 V_{h} \cos \left(\frac{\pi b}{N+1}\right), \quad b=1, \ldots, N .
$$

If $V_{c}$ is the spin independent coupling between the molecule and the edge buffer site, then the tunneling matrix elements in Eq. (33) are

$$
t_{b}=V_{c} \sqrt{\frac{2}{N+1}} \sin \left(\frac{\pi b}{N+1}\right), \quad b=1, \ldots, N .
$$



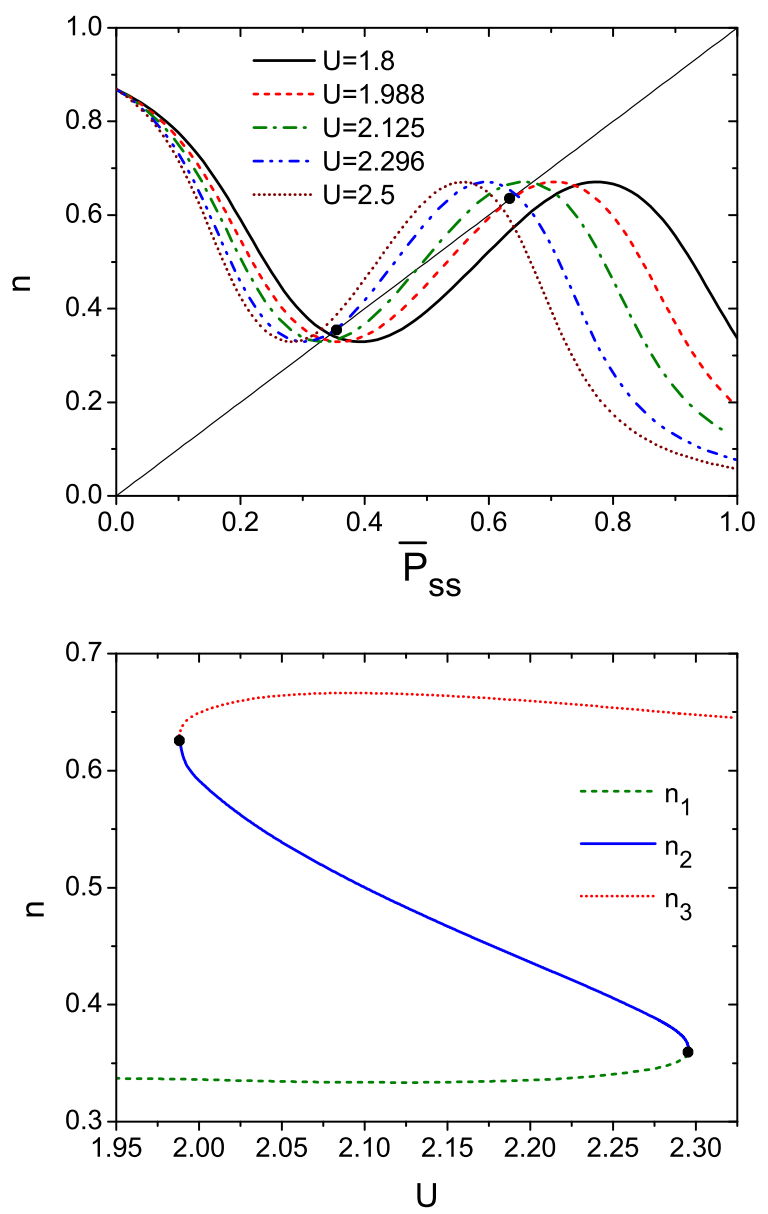

FIG. 2: Upper panel: The graphical solution of stationary nonequilibrium Hartree-Fock equations (38) for different valued of $U$. Lower panel: the fixed point electron densities $n_{k}=\bar{P}_{s s}^{(k)}(k=1,2,3)$ as functions of $U$. Black circles indicates saddle-node bifurcation points.

The parameter $\gamma_{b}$ in Lindblad master equation is taken to be equal to the distance between neighbor energy levels in the buffer zones, i.e., $\gamma_{b}=\varepsilon_{b}-\varepsilon_{b+1}$.

In our calculations we use the same parameters as in [2]. Namely, $V_{h}=-0.5, V_{c}=-0.35$, and the molecular orbital energy is $\varepsilon_{0}=0$. The buffer on-site energies are shifted by the applied voltage bias $\left(V=V_{L}-V_{R}\right)$ : $\epsilon_{L, R}=0.3+V_{L, R}$, where $V_{L}=1.5$ and $V_{R}=0.0$. The leads are half-filled so that the Fermi levels of lead $L, R$ are positioned at $\epsilon_{L, R}$. The inverse temperature is $\beta=90$. We use $N=400$ and this choice will be justified below. Here we keep $U$ as a variable quantity and illustrate how the number and properties of nonequilibrium fixed points depend on the strength of electron-electron interaction.

The time-dependent Hartree-Fock dynamics for the model Hamiltonian (33) is fully characterized by the fol- lowing (spin independent) single-particle density matrix:

$$
\begin{aligned}
& P_{s s}(t)=\left\langle a_{\sigma}^{\dagger} a_{\sigma}\right\rangle_{t}, \\
& P_{s b}(t)=\left\langle a_{b \sigma}^{\dagger} a_{\sigma}\right\rangle_{t}, P_{b b^{\prime}}(t)=\left\langle a_{b^{\prime} \sigma}^{\dagger} a_{b \sigma}\right\rangle_{t} .
\end{aligned}
$$

To find the fixed point densities, $\bar{P}_{\alpha \beta}$, we solve the nonequilibrium stationary Hartree-Fock equations (i.e. the system of equations (27) with the time-derivatives of the density matrix set to zero):

$$
\begin{aligned}
& \sum_{b} t_{b}\left(\bar{P}_{s b}-\bar{P}_{b s}\right)=0, \\
& t_{b} \bar{P}_{s s}+\left(\varepsilon_{0}+U \bar{P}_{s s}-E_{b}^{*}\right) \bar{P}_{s b}-\sum_{b^{\prime}} t_{b^{\prime}} \bar{P}_{b^{\prime} b}=0, \\
& t_{b} \bar{P}_{s b^{\prime}}-t_{b^{\prime}} \bar{P}_{b s}-\left(E_{b}-E_{b^{\prime}}^{*}\right) \bar{P}_{b b^{\prime}}=2 i \delta_{b b^{\prime}} f_{b} \gamma_{b} .
\end{aligned}
$$

These Hartree-Fock equations are nonlinear with respect to the fixed point electron density in the molecule, $n=$ $\bar{P}_{s s}$.

By numerical solution of the Hartree-Fock equations we have found that there is a range of the Coulomb interaction strength parameters, $U$, for which Eq. (38) have multipole fixed point solutions $\bar{P}_{\alpha \beta}^{(k)}(k=1,2, \ldots)$. In the upper panel of Fig. 2 we show the graphical solution of Eq. (38) for the different values of $U$. In this plot the crossings of the straight and curved lines give molecular densities $n=\bar{P}_{s s}$ corresponding to different fixed point solutions of Eq. (38). We see that three cases are possible depending on the value of $U$ : when $1.988<U<2.296$ there exist three fixed points; at the ends of the interval we have two fixed points; and outside the interval there is only one fixed point. Hereafter we will number fixed point molecular densities in ascending order, i.e., $n_{1} \leq n_{2} \leq n_{3}$.

In the lower panel of Fig. 2 we show how the molecular densities $n_{k}$ depend on the Coulomb interaction strength. The "middle" density $n_{2}$ demonstrates the strong $U$ dependency and it exists only when $1.988<U<2.296$. The fixed point corresponding to $n_{2}$ collides with that corresponding to $n_{3}\left(n_{1}\right)$ and annihilate it at the left (right) end of this interval for $U$. This is so-called saddlenode bifurcation point [12] (indicated by black circles in Fig. (2).

Let us understand which of these three fixed point solutions are asymptotically stable (i.e., nonequilibrium steady states). For this purpose we construct the stability matrix (32) for our model Hamiltonian. The only non-zero matrix elements of the stability matrix are

$$
\begin{aligned}
& A_{s s, s b}=-i t_{b}, A_{s s, b s}=i t_{b}, A_{s b, b^{\prime} b}=i t_{b^{\prime}} \\
& A_{s b, s s}=-i\left(t_{b}+U \bar{P}_{s b}\right), A_{s b, s b}=-i\left[\left(\varepsilon_{0}+U \bar{P}_{s s}\right)-E_{b}^{*}\right] \\
& A_{b s, s s}=i\left(t_{b}+U \bar{P}_{b s}\right), A_{b s, b s}=i\left[\left(\varepsilon_{0}+U \bar{P}_{s s}\right)-E_{b}\right] \\
& A_{b s, b b^{\prime}}=-i t_{b^{\prime}}, A_{b b^{\prime}, s b^{\prime}}=i t_{b}, A_{b b^{\prime}, b s}=-i t_{b^{\prime}} \\
& A_{b b^{\prime}, b b^{\prime}}=-i\left(E_{b}-E_{b^{\prime}}^{*}\right) .
\end{aligned}
$$

The dimension of the stability matrix is $(1+2 N)^{2}$ and it is sparse. For a given fixed point $\bar{P}_{\alpha \beta}^{(k)}$ we compute numerically first few eigenvalues of the corresponding stability 
TABLE I: Self-consistent electron densities on the molecule $n_{k}=\bar{P}_{s s}^{(k)},(k=1,2,3)$ for nonequilibrium fixed points computed by the Lindblad kinetic equation with different size of the buffer zones $(N=200,400,600)$ and by the NEGF method.

\begin{tabular}{|l||c|c|c||c|c|c||c|c|c||c|c|c|}
\hline \multicolumn{1}{|c||}{$\mathrm{U}$} & \multicolumn{3}{c||}{$N=200$} & \multicolumn{3}{c||}{$N=400$} & \multicolumn{3}{c||}{$N=600$} & \multicolumn{3}{c|}{ NEGF } \\
\cline { 2 - 11 } & $n_{1}$ & $n_{2}$ & $n_{3}$ & $n_{1}$ & $n_{2}$ & $n_{3}$ & $n_{1}$ & $n_{2}$ & $n_{3}$ & $n_{1}$ & $n_{2}$ & $n_{3}$ \\
\hline 2.0 & 0.34 & 0.60 & 0.64 & 0.34 & 0.59 & 0.65 & 0.34 & 0.59 & 0.65 & 0.33 & 0.58 & 0.66 \\
2.05 & 0.34 & 0.54 & 0.66 & 0.33 & 0.54 & 0.67 & 0.33 & 0.54 & 0.67 & 0.33 & 0.54 & 0.67 \\
2.10 & 0.34 & 0.50 & 0.66 & 0.33 & 0.50 & 0.67 & 0.33 & 0.50 & 0.67 & 0.33 & 0.50 & 0.67 \\
2.15 & 0.34 & 0.47 & 0.66 & 0.33 & 0.47 & 0.66 & 0.33 & 0.47 & 0.67 & 0.33 & 0.47 & 0.67 \\
2.20 & 0.34 & 0.43 & 0.66 & 0.34 & 0.44 & 0.66 & 0.33 & 0.44 & 0.66 & 0.33 & 0.44 & 0.66 \\
2.25 & 0.35 & 0.40 & 0.65 & 0.34 & 0.41 & 0.65 & 0.34 & 0.41 & 0.66 & 0.33 & 0.41 & 0.66 \\
\hline
\end{tabular}

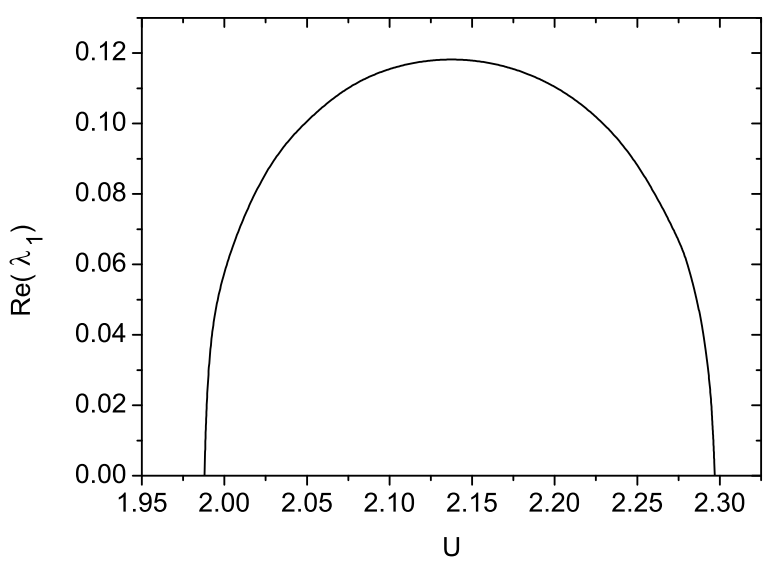

FIG. 3: The dependence of $\operatorname{Re}\left(\lambda_{1}\right)$ as a function of $U$ for the unstable nonequilibrium fixed point.

matrix with the largest real parts $\left(\operatorname{Re}\left(\lambda_{1}\right) \geq \operatorname{Re}\left(\lambda_{2}\right) \geq\right.$ ...). For this aim we used the ARPACK nonsymmeteric sparse eigenvalue solver. [18] We find that the stability matrices resulting from $\bar{P}_{\alpha \beta}^{(1)}$ and $\bar{P}_{\alpha \beta}^{(3)}$ do not have eigenvalues with positive real parts, therefore the corresponding fixed points are stable. Contrary, the stability matrix resulting from $\bar{P}_{\alpha \beta}^{(2)}$ always has at least one eigenvalue $\lambda_{1}$ with a positive real part, i.e., the corresponding fixed point is unstable.

In Fig. 3 we plot $\operatorname{Re}\left(\lambda_{1}\right)$ as a function of $U$ for the unstable fixed point $\bar{P}_{\alpha \beta}^{(2)}$. The obtained curve is symmetric with respect to $U=2.143$ where $\operatorname{Re}\left(\lambda_{1}\right)$ reaches its maximum value. At $U=1.988$ and $U=2.296$, when the saddle-node bifurcation points are reached, eigenvalue $\lambda_{1}$ becomes zero. Thus, our stability analysis is consistent with the dynamical observations of [2] that the "middle" fixed point can not be reached by time-dependent prop-

\section{CONCLUSIONS}

We have presented a theoretical method to perform a stability analysis of multiple nonequilibrium fixed points, agation of the NEGF Kadanoff-Baum equation. We do not observe purely imaginary eigenvalues of the stability matrix, therefore we rule out the existence of periodically oscillating fixed points in non-equilibrium Hartree-Fock approximation for the Anderson model.

In Table III we compare the calculated $(N=$ 200, 400, 600) fixed point densities of the molecule with the exact Hartree-Fock densities obtained by the NEGF method. The latter are the solutions of the following equation: [19]

$$
n=\frac{1}{\pi} \int d \omega \frac{\Gamma_{L}(\omega) f_{L}(\omega)+\Gamma_{R}(\omega) f_{R}(\omega)}{(\omega-\varepsilon-U n-\Lambda(\omega))^{2}+(\Gamma(\omega))^{2}} .
$$

Here $f_{L, R}(\omega)=\left[1+e^{\left(\omega-\mu_{L, R}\right) / T}\right]^{-1}$ is the Fermi-Dirac electron distribution in the left and right electrodes, and $\Lambda=\Lambda_{L}+\Lambda_{R}, \Gamma=\Gamma_{L}+\Gamma_{R}$ are the real and imaginary parts of the leads self-energy. For the tight binding electrodes, that we consider, the self-energy is given by

$$
\begin{aligned}
& \Sigma_{L, R}(\omega)=\Lambda_{L, R}(\omega)-i \Gamma_{L, R}(\omega) \\
& =\frac{V_{h}^{2}}{2 V_{c}^{2}}\left\{\begin{array}{cc}
\omega_{L, R}-\sqrt{\omega_{L, R}^{2}-4 V_{c}^{2}}, & \omega_{L, R}>2\left|V_{c}\right| \\
\omega_{L, R}+\sqrt{\omega_{L, R}^{2}-4 V_{c}^{2}}, & \omega_{L, R}<-2\left|V_{c}\right| \\
\omega_{L, R}-i \sqrt{4 V_{c}^{2}-\omega_{L, R}^{2}}, & \left|\omega_{L, R}\right|<2\left|V_{c}\right|
\end{array}\right.
\end{aligned}
$$

where $\omega_{L, R}=\omega-\epsilon_{L, R}$. As seen from the table the larger $N$ the better our method reproduce the exact result. For $N=400$ our method quite well reproduce the exact result (the maximum deviation from the exact results is under $2 \%$ ), therefore our choice of the size of the buffer zone is justified and makes our Lindblad-type kinetic equation numerically exact for electron transport calculations. which appear in self-consistent electronic transport calculations. We employed the Lindblad kinetic equation and 
underlying approximations of this kinetic equation were alleviated by the use of explicit buffer zone between the molecule and electronic electrodes. Nonequilibrium fixed point were obtained from the self-consistent solution of the kinetic equation in stationary limit. The asymptotic stability of these fixed points were studied by linearizing of the nonlinear kinetic equation. We obtained the nonHermitian stability matrix from the linearized kinetic equation and analyzed its spectrum. If real parts of all eigenvalues of the stability matrix are negative, then the fixed point is asymptotically stable as $t \rightarrow \infty$ and it can be regarded as steady state. If at least one eigenvalue has positive real part, the solution becomes unstable and can not be the steady state. We obtained the explicit form of the stability matrix in Hartree-Fock and adiabatic timedependent DFT approximations. The method was applied to out of equilibrium Anderson model which yields three nonequilibrium fixed points under certain choice of parameters in nonequilibrium Hartree-Fock approximation. We performed the stability analyse of these fixed points and demonstrated that one fixed point is asymptotically unstable whereas the other fixed points correspond to physical steady states.

\section{Acknowledgments}

This work has been supported by the Francqui Foundation, Belgian Federal Government under the Interuniversity Attraction Pole project NOSY and Programme d'Actions de Recherche Concertée de la Communauté francaise (Belgium) under project "Theoretical and experimental approaches to surface reactions".

\section{Appendix A: Lindblad kinetic equation for embedded molecule}

Let us begin with the Liouville equation for the total density matrix $\chi(t)$

$$
i \dot{\chi}(t)=[\mathcal{H}, \chi(t)] .
$$

We partition the Hamiltonian (7) into two parts:

$$
\begin{gathered}
h=H_{M}+H_{E}+H_{B}+H_{S B} \\
v=H_{E B}
\end{gathered}
$$

In the interaction representation the Liouville equation becomes

$$
\begin{aligned}
\dot{\chi}_{I}(t) & =\frac{1}{i}\left[v_{I}(t), \chi_{I}(0)\right] \\
& -\int_{0}^{t} d \tau\left[\left[v_{I}(t),\left[v_{I}(t-\tau), \chi_{I}(t-\tau)\right]\right],\right.
\end{aligned}
$$

where $\chi_{I}(t)=e^{i h t} \chi(t) e^{-i h t}$ and $v_{I}(t)=e^{i h t} v e^{-i h t}$. Now we introduce the density matrix for embedded system (molecule plus buffer) by tracing out the environment degrees of freedom

$$
\rho(t)=\operatorname{Tr}_{E} \chi(t)
$$

We assume that the total density matrix can be factorized and environmental degrees of freedom propagate in time as there were no interaction with the buffer (Born approximation):

$$
\chi_{I}(t)=\rho_{I}(t) \rho_{E} .
$$

The density matrix for the environment is taken in the grand canonical ensemble form

$$
\rho_{E} \sim e^{-\sum_{\sigma, k \in L} \beta_{L}\left(\varepsilon_{k}-\mu_{L}\right) a_{k \sigma}^{\dagger} a_{k \sigma}} e^{-\sum_{\sigma, k \in R} \beta_{R}\left(\varepsilon_{k}-\mu_{R}\right) a_{k \sigma}^{\dagger} a_{k \sigma}} .
$$

Setting $\mu_{L} \neq \mu_{R}$ and/or $\beta_{L} \neq \beta_{R}$ in the environment, we drive the system out of equilibrium.

After tracing, Eq. A4 becomes

$$
\dot{\rho}_{I}(t)=-\int_{0}^{t} d \tau \operatorname{Tr}_{E}\left[\left[v_{I}(t),\left[v_{I}(t-\tau), \rho_{I}(t-\tau) \rho_{E}\right]\right]\right.
$$

Here, we have assumed that $\operatorname{Tr}_{E}\left[v_{I}(t), \chi_{I}(0)\right]=0$.

Using the explicit expression for the Hamiltonian $h$ one can easily demonstrate that

$$
\begin{aligned}
e^{i h t} a_{k \sigma} e^{-i h t} & =e^{-i \varepsilon_{k} t} a_{k \sigma}, \\
e^{i h t} a_{b \sigma} e^{-i h t} & =e^{-i \varepsilon_{b} t} a_{b \sigma}+O\left(1 / N_{B}\right) .
\end{aligned}
$$

where $N_{B}$ is the number of discrete single particle levels (i.e. the size) of the buffer zone. Therefore by choosing the large enough buffer zone, we may assume that buffer single-particle states evolve in time as free states. As a result, $v_{I}(t)$ takes the form

$$
v_{I}(t)=\sum_{\sigma b k}\left(v_{b k}(t) a_{b \sigma}^{\dagger} a_{k \sigma}+h . c .\right)
$$

where $v_{b k}(t)=v_{b k} e^{i\left(\varepsilon_{k}-\varepsilon_{b}\right) t}$.

Now, the kinetic equation for the embedded system density matrix becomes 


$$
\begin{array}{r}
\dot{\rho}_{I}(t)=-\int_{0}^{t} d \tau \sum_{\sigma k b b^{\prime}}\left\{v_{b k}(t) v_{b^{\prime} k}^{*}(t-\tau)\left[\left(1-f_{k}\right) a_{b \sigma}^{\dagger}\left[a_{b^{\prime} \sigma}, \rho_{I}(t-\tau)\right]-f_{k}\left[a_{b^{\prime} \sigma}, \rho_{I}(t-\tau)\right] a_{b \sigma}^{\dagger}\right]+\right. \\
\left.v_{b k}^{*}(t) v_{b^{\prime} k}(t-\tau)\left[f_{k} a_{b \sigma}\left[a_{b^{\prime} \sigma}^{\dagger}, \rho_{I}(t-\tau)\right]-\left(1-f_{k}\right)\left[a_{b^{\prime} \sigma}^{\dagger}, \rho_{I}(t-\tau)\right] a_{b \sigma}\right]\right\} .
\end{array}
$$

Here $f_{k \in L / R}=\operatorname{Tr}_{E}\left(\rho_{E} a_{k \sigma}^{\dagger} a_{k \sigma}\right)=\left[1+e^{\beta_{L / R}\left(\varepsilon_{k}-\mu_{L / R}\right)}\right]^{-1}$ Assuming that the environment relaxation time is very fast we can extend the integration range to $+\infty$ and $\rho(t-\tau) \simeq \rho(t)$ (Markov approximation). Finally, in the rotating wave approximation rapidly oscillating terms proportional to $\exp \left[i\left(\varepsilon_{b}-\varepsilon_{b}^{\prime}\right) t\right]$ for $\varepsilon_{b} \neq \varepsilon_{b}^{\prime}$ are neglected. Then, the kinetic equation (A11) becomes the standard Lindblad type master equation (16).

The obtained Lindblad master equation describes the time evolution of the open embedded system preserving the probability and the positivity of the density matrix. Open boundary conditions are introduced via nonHermitian dissipative part of Eq.(16), $\hat{\Pi} \rho(t)$, which represents the influence of environment on the system. The applied bias potential enters into Eq.(16) via fermionic occupation numbers $f_{b}(b \in L, R)$ which depend on the chemical potential in the light and right electrodes.

\section{Appendix B: Stability matrix for nonequilibrium self-consistent DFT electron transport calculations}

Leaving apart the conceptual questions about the use of ground state DFT for self-consistent electronic transport calculations, we can say that the only difference in practical computations between nonequilibrium timedependent Hartree-Fock discussion (Section II) and adiabatic time-dependent Kohn-Sham DFT is that the timedependent Fock matrix (24) becomes

$$
F_{n m}(t)=T_{n m}+\int d \mathbf{r} \phi_{n}(\mathbf{r}) v_{K S}^{\sigma}(\mathbf{r}, t) \phi_{m}(\mathbf{r}) .
$$

Here

$$
v_{K S}^{\sigma}(\mathbf{r}, t)=\int d \mathbf{r}^{\prime} \frac{\rho\left(\mathbf{r}^{\prime}, t\right)}{\left|\mathbf{r}-\mathbf{r}^{\prime}\right|}+v_{x c}^{\sigma}[\rho](\mathbf{r}, t),
$$

where $v_{x c}^{\sigma}[\rho](\mathbf{r}, t)$ is the exchange-correlation potential. Then the system of equations (27) and (29) remains the same, but the steady state Fock matrix (30) becomes

$$
\bar{F}_{n m}=T_{n m}+\int d \mathbf{r} \phi_{n}(\mathbf{r}) v_{K S}^{\sigma}[\bar{\rho}](\mathbf{r}) \phi_{m}(\mathbf{r})
$$

and variation (31) is

$$
\delta F_{n m}(t)=\int d \mathbf{r} \phi_{n}(\mathbf{r}) \delta v_{K S}^{\sigma}(\mathbf{r}, t) \phi_{m}(\mathbf{r}),
$$

where $\delta v_{K S}^{\sigma}$ is given by the standard expression of linear response time-dependent DFT

$$
\begin{aligned}
& \delta v_{K S}^{\sigma}(\mathbf{r}, t)=\int d \mathbf{r}^{\prime} \frac{\delta \rho\left(\mathbf{r}^{\prime}, t\right)}{\left|\mathbf{r}-\mathbf{r}^{\prime}\right|} \\
& +\sum_{\sigma^{\prime}} \int d \mathbf{r}^{\prime} f_{x c}\left(\sigma \mathbf{r}, \sigma^{\prime} \mathbf{r}^{\prime}\right) \delta \rho_{\sigma^{\prime}}\left(\mathbf{r}^{\prime}, t\right) .
\end{aligned}
$$

The exchange-correlation response kernel $f_{x c}\left(\sigma \mathbf{r}, \sigma^{\prime} \mathbf{r}^{\prime}\right)$ is given in the usual adiabatic approximation, [20, 21] i.e., the exchange-correlation contribution is taken to be simply the second derivative of the static ground state exchange-correlation energy $E_{x c}$ with respect to the fixed point spin density $\bar{\rho}_{\sigma}(\mathbf{r})$ :

$$
f_{x c}\left(\sigma \mathbf{r}, \sigma^{\prime} \mathbf{r}^{\prime}\right)=\delta\left(\mathbf{r}-\mathbf{r}^{\prime}\right) \frac{\delta E_{x c}[\bar{\rho}]}{\delta \bar{\rho}_{\sigma^{\prime}}\left(\mathbf{r}^{\prime}\right) \delta \bar{\rho}_{\sigma}(\mathbf{r})}
$$

Therefore, the stability analysis of nonequilibrium fixed points for self-consistent DFT electronic transport calculations can be readily performed within standard adiabatic time-dependent density functional response theory. $22,24]$
[1] A. Dhar, Advances in Physics 57, 457 (2008).

[2] A.-M. Uimonen, E. Khosravi, G. Stefanucci, S. Kurth, R. van Leeuwen, and E. K. U. Gross, Journal of Physics: Conference Series 220, 012018 (2010).

[3] A. S. Alexandrov, A. M. Bratkovsky, and R. S. Williams, Phys. Rev. B 67, 075301 (2003).

[4] M. Galperin, M. A. Ratner, and A. Nitzan, Nano Letters 5, 125 (2005).

[5] C. Negre, P. Gallay, and C. G. Sánchez, Chem. Phys. Lett. 460, 220 (2008).
[6] J. Taylor, H. Guo, and J. Wang, Phys. Rev. B 6324, 245407 (2001).

[7] M. Brandbyge, J. L. Mozos, P. Ordejon, J. Taylor, and K. Stokbro, Phys. Rev. B 65, 165401 (2002).

[8] Y. Q. Xue, S. Datta, and M. A. Ratner, Chem. Phys. 281, 151 (2002).

[9] S. H. Ke, H. U. Baranger, and W. T. Yang, Phys. Rev. B 70, 085410 (2004).

[10] C. G. Sánchez, M. Stamenova, S. Sanvito, D. R. Bowler, A. P. Horsfield, and T. N. Todorov, J. Chem. Phys. 124, 
214708 (2006).

[11] A. S. Mikhailov and A. Y. Loskutov, Foundations of Synergetics II: Complex Patterns (Springer, Berlin/Heidelberg, 1996).

[12] S. H. Strogatz, Nonlinear Dynamics And Chaos: With Applications To Physics, Biology, Chemistry, And Engineering (Studies in Nonlinearity), 1st ed., (Perseus Books Group, New York, 1994).

[13] H. P. Breuer and F. Petruccione, The Theory of Open Quantum Systems (Oxford University Press, Oxford, 2002).

[14] A. A. Dzhioev and D. S. Kosov, J. Chem. Phys. 134, 044121 (2011).

[15] A. A. Dzhioev and D. S. Kosov, J. Chem. Phys. 134, 154107 (2011).

[16] E. J. McEniry, D. R. Bowler, D. Dundas, A. P. Horsfield, C. G. Sánchez, and T. N. Todorov, Journal of Physics: Condensed Matter 19, 196201 (2007).

[17] S. Kurth, G. Stefanucci, E. Khosravi, C. Verdozzi, and E. K. U. Gross, Phys. Rev. Lett. 104, 236801 (2010).
[18] R. B. Lehoucq, D. C. Sorensen, and C. Yang, ARPACK User's Guide: Solution of Large-Scale Eigenvalue Problems With Implicitly Restarted Arnoldi Methods (Software, Environments, Tools) (Society for Industrial \& Applied Math, Philadelphia, 1998).

[19] H. Haug and A. Jauho, Quantum Kinetics in Transport and Optics of Semiconductors (Springer, Berlin/Heidelberg, 2010).

[20] E. K. U. Gross and W. Kohn, Adv. Quantum Chem. 21, 255 (1990).

[21] M. E. Casida, in Recent Advances in Density-Functional Methods, edited by D. P. Chong (World Scientific, Singapore, 1995), p. 155.

[22] N. L. Doltsinis and M. Sprik, Chem. Phys. Lett. 330, 563 (2000).

[23] F. Furche and R. Ahlrichs, J. Chem. Phys. 117, 7433 (2002).

[24] N. L. Doltsinis and D. S. Kosov, J. Chem. Phys. 122, 144101 (2005). 